\title{
Design and testing of piezoelectric flight control actuators for hard-launch munitions
}

\author{
Ron Barrett*a and Gary Lee ${ }^{b}$ \\ ${ }^{a}$ Faculty of Aerospace Engineering, Kluyverweg 1,Technical University Delft, 2629HS Netherlands \\ ${ }^{b}$ President, Lutronix Corporation, 13627 Portofino Drive, Del Mar, California 92014
}

\begin{abstract}
A new technique is presented for designing actuators for guided hard-launch adaptive munitions by using actuator and substrate strain limits, static analysis methods and matching the local actuator strains along its length by varying the width. This Load-Matched design technique leads to an exponential area distribution as a function of length which is contrasted against the conventional rectangular actuator shapes that have been used in all adaptive hard-launch munitions up till now. To demonstrate the viability of this new Load-Matched actuator design, ten $600 \mathrm{mg}, 100 \mathrm{~mm}$ long rectangular and ten identical mass and length, exponentially shaped, Load-Matched actuator specimens were designed and built to withstand the maximum possible accelerations. Predicted design static strain distributions are presented along with limits, showing that rectangular actuators exhibit a strong strain peak at the root while Load-Matched actuators have a much more even distribution and a gentle maximum near the middle. Shock table testing showed that the rectangular specimens were predicted to fail at 3,500g's, but survived acceleration levels $9.5-12 \%$ higher than expected $(3,833$ to $3,931 \mathrm{~g}$ 's). The exponentially shaped Load-Matched actuators proved that they could withstand shocks from 17 to $21 \%$ over the predicted failure acceleration level of $8,000 \mathrm{~g}$ 's $(9,377$ to $9,670 \mathrm{~g}$ 's).
\end{abstract}

Keywords: Piezoelectric Adaptive Munition Cannon Shell Bullet Flight Control

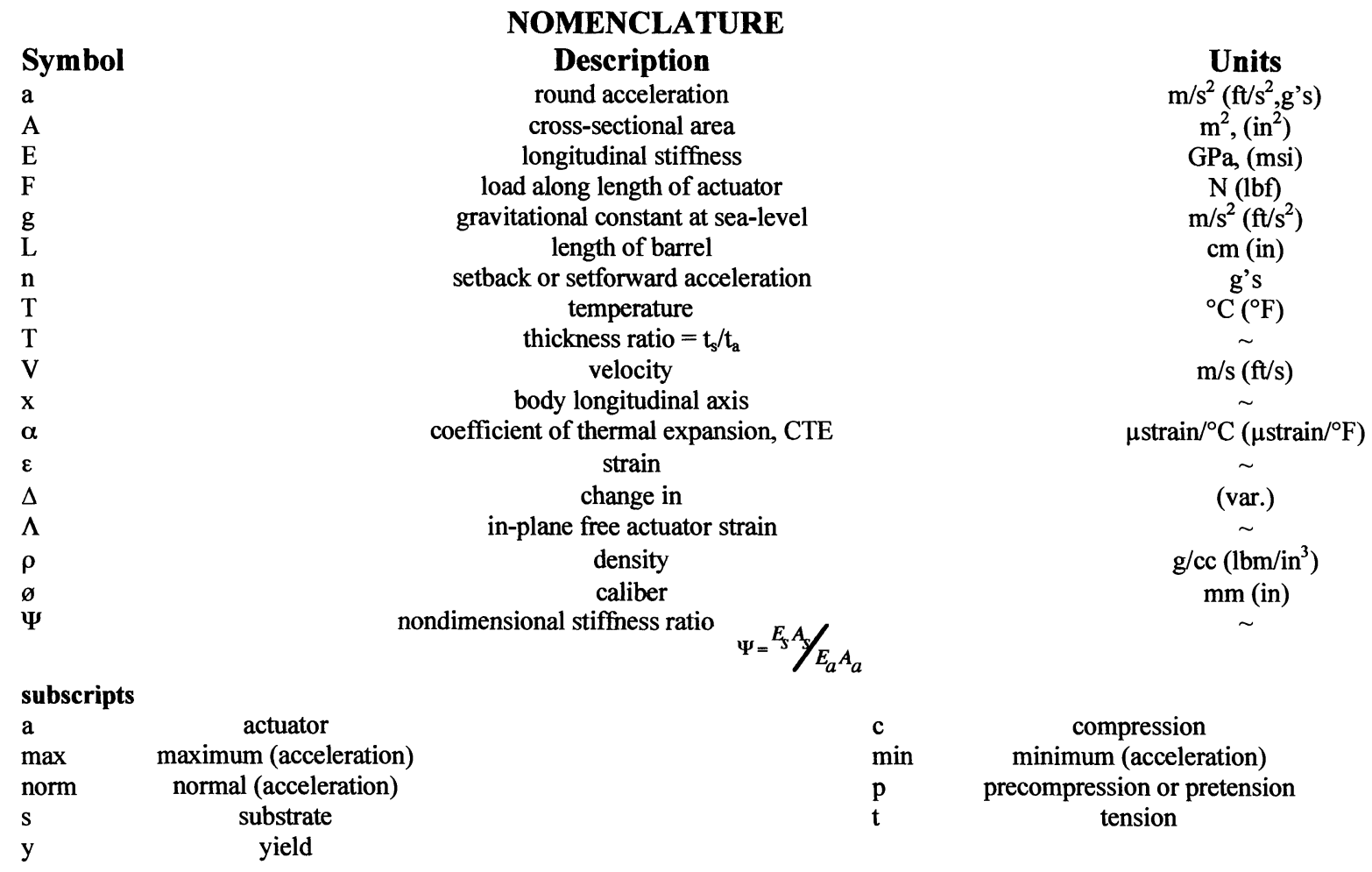




\section{INTRODUCTION}

Delivering projectiles to specific locations has been central to the field of gunnery since the first shot was fired more than a thousand years ago. Improvements in accuracy have primarily stemmed from tighter manufacturing tolerances and greater knowledge and implementation of effective passive methods like spin stabilization. Although modern investigators have worked to limit several of these error sources including charge-induced range deviations and tip-off errors from barrel dynamics, some major sources remain a challenge for current technology to sense and/or control ${ }^{1,2}$ Chief among these sources are atmospheric disturbances over the flight path. As range increases, the necessity of correcting for comparatively unpredictable winds becomes more and more important. In larger rounds $(81 \mathrm{~mm}$ and up in caliber), spatial errors are easily corrected through the use of conventional electromagnetic actuators. Indeed, more than 30 years ago the Copperhead antitank missile was successfully designed, developed and fielded using such actuators. ${ }^{3}$ More modern programs have similarly included guidance on $105,120 \mathrm{~mm}$ and larger rounds, including the XM-982, STAFF, ERGM and Competent Munitions programs to name a few. ${ }^{4-7}$ Others have pushed the boundary down to the $81 \mathrm{~mm}$ mark with the Merlin guided mortar rounds. ${ }^{8}$

Although flight control and guidance packages using current technology are well suited to such larger caliber weapons, there remains a wide array of weapons below this range which could make good use of similar systems. One of the early efforts to guide smaller cannon shells was conducted in the early 1980's in the Sergeant York program. Although the program was stopped, several pioneering steering mechanisms were conceived and prototyped on $40 \mathrm{~mm}$ rounds. ${ }^{9}$

Since that time, some new technologies have come to the fore which have pushed the envelope for possible round guidance down in caliber. Chief among them is the field of adaptive structures. Papers from the early 1990's describe the successful integration of these materials in missile and munitions fins on the scale of $10 \mathrm{~cm}^{10-12}$ In September of 1994, the first UAV to use adaptive materials for all flight control, Mothra, took to the air, demonstrating low weight, high efficiency and bandwidths which far exceeded conventional electromagnetic servos of similar weight and volume. ${ }^{13}$

Following the success of Mothra, investigators branched out into munitions of larger caliber, demonstrating successful full flight control of air-to-ground area weapons and vernier control of penetrator weapons. ${ }^{14,15}$ The Smart Compressed Reversed Adaptive Munition (SCRAM) and Weapon Integration and Design Technology (WIDT) programs used piezoelectric actuator elements which were tailored to weapon geometries to minimize weight, volume and cost impact. Both programs demonstrated flight control actuator shrinkage to such a level that the actuators could be packed within aerodynamic surfaces, thereby alleviating the necessity of penetrating the outer mold lines of the fuselage. Because this actuator class showed such great promise in these weapons and was so amenable to integration on smaller weapons a new effort was started on hard-launched munitions. In the summer of 1995, the first Barrel-Launched Adaptive Munition (BLAM) was conceived and developed. This munition employed piezoelectric tendon actuators to pivot a conical nose of a conical round for round steering and acted as the earliest proof-of-principle work on adaptive hard-launched adaptive munitions. ${ }^{16-19}$ Figure 1 shows the internal arrangement of the round, including two pairs of orthogonal piezoelectric tendons, a nose pivot, notional seeker assembly and a grapple for wind tunnel testing.

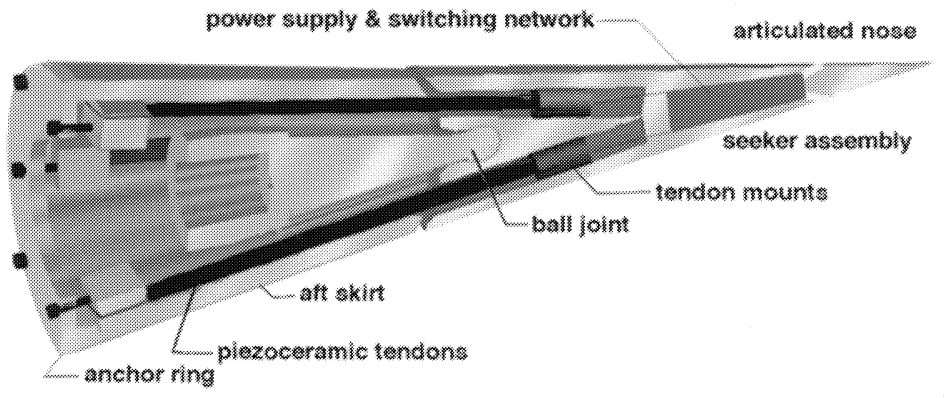

Fig. 1 Barrel-Launched Adaptive Munition (BLAM) internal arrangement ${ }^{17}$

Although it never lead to a fielded system for aerial gunnery (as intended), several important principles were discovered during the course of the program. By drawing upon work performed on missile fins in 1990 and 1991, the principle of precompression through coefficient of thermal expansion mismatch between substrate and actuators was greatly expanded. ${ }^{11,12}$ A complete study of suitable substrate materials was made and used as a basis upon which to form fundamental laminated plate theory estimations which could be used to rapidly lay out different geometries of actuators. ${ }^{16}$ At the same time, the aerodynamics of articulated conical shells was also being researched and showed that significant flight path deviations could be commanded via finite articulations of under $1 \mathrm{deg} .{ }^{18,19}$ 
Since these early days, numerous other subscale adaptive munition programs have slowly matured the technology to the point of viability. ${ }^{20-22}$ Current advances in actuator technology have lead to yet further quantum leaps in capability over the state-of-the-art in terms of setback acceleration resistance, bandwidth, weight, volume and flight control authority. ${ }^{23}$ Although nearly all of these modern efforts are subject to various disclosure restrictions, it is important for the technical community to understand that they are underway, have been highly successful and are rapidly evolving. Accordingly, the purpose of this paper is to introduce the technical community to the fundamental design principles behind this new class of enabling elements and deliver basic experimental data on a new actuator design which demonstrates their suitability as flight control actuators for subscale hard-launched munitions.

\section{GENERAL DESIGN CONDITIONS FOR HARD-LAUNCH ACTUATOR SYSTEMS}

Hard-launch munition actuator systems are designed to withstand enormous accelerations which are endured during and just after launch, followed by comparatively negligible accelerations in free flight. Compounding these conditions are a myriad of other factors which must be included to survive and be viable in the military environment. The short summary below illustrates some of these challenges.

\subsection{Setback Accelerations}

Setback accelerations are the dominant environmental condition of most hard-launched structures and are experienced from the time of initial charge detonation till just after the round exits the barrel. They strongly influence structural layout and material choices and are the driving condition behind length limitations of hard-launched actuators. Although munitions designers typically use exacting profiles which are specific to a gun, round, muzzle velocity and charge type combination to predict peak setback accelerations, some basic boundaries can be gleaned from fundamental physics and empirical trends for the purposes of first-order design. If one assumes a constant acceleration along the length of a barrel, a round starting from 0 and exiting at a finite muzzle velocity, then this "minimum acceleration" or "ideal traveling charge" profile will yield a lower bound below which it is not possible to go below:

$$
a_{\min }=V_{\text {muzzle }}^{2} / L_{\text {barrel }}
$$

Because there is no similar upper bound which can be obtained by simple physics, generalized trends from interior ballistic profiles can be obtained. By examining the acceleration profiles of instrumented rounds like the Hypervelocity Weapon System, a rough upper bound can be gleaned for initial design purposes. ${ }^{24}$

$$
a_{\text {peak }} \cong 1.45 V_{\text {muzzle }}^{2} / L_{\text {barrel }}
$$

For larger caliber artillery rounds which are currently fielded, setback accelerations on the order of $5,000-30,000$ g's are typical. The ERGM projectile is typical of the current families of guided 5 " $(127 \mathrm{~mm})$ cannon shells and is designed for $12,000 \mathrm{~g}$ 's of setback acceleration while the LCCM projectiles are designed to withstand $15,000 \mathrm{~g}$ 's. ${ }^{1,25}$

\subsection{Setforward, Balloting and Ringing}

Although secondary to setback accelerations, setforward accelerations have extremely detrimental effects on all hard-launch round components and subsystems. Setforward accelerations are typically induced as the supersonic round exits the barrel into essentially still air. This typically causes a comparatively large drag force which decelerates most rounds with a pulse of approximately one order of magnitude lower than the setback acceleration. Setforward accelerations are often experienced as the round passes through a muzzle flash deflector and/or during sabot separation. Setforward accelerations are the principal loads which induce buckling and end crush-out failure modes of adaptive actuators. Reference 25 lists the design setforward accelerations for the ERGM round to be 2,500g's.

Balloting is just as detrimental to many internal round structures as accelerations are applied to the round in a direction which is orthogonal to the longitudinal axis. Balloting is typically induced by both barrel flexure and internal dynamics between the barrel and the round as it travels from breech to muzzle, intermittently coming in contact with both the sides of the barrel and asymmetric boundary layer/shock structures. Reference 25 lists the balloting design accelerations as 2,500g's for the ERGM round.

Ringing is essentially an after effect of extremely high accelerations and is principally caused by gross round and internal structure dynamics within the round following launch. Although ringing accelerations are typically an order of magnitude still below setforward accelerations, they tend to fatigue components in general and cold work metal parts, 
including solder joints and wires often to failure. Although ringing is a real, observable effect on hard launched munitions, it is even more pronounced during shock table tests because of plate slap dynamics. Because shock table ringing effects are often more robust and long lived than what is experienced in flight, many ballisticians will qualify a given package on a shock table to a peak setback acceleration level, then clear it for launch knowing that it has been exposed to a more violent environment than will be experienced tactically.

\subsection{Rotational Accelerations}

Most of the hard-launch munitions which are in use today employ spin to provide passive stabilization as unspun rounds would otherwise be inherently longitudinally unstable. The principal mechanism for inducing spin into a round is rifling in the barrel. Such rifling induces acceleration rates on the order of several hundred thousand rad/s2. From Ref. 1, the LCCM design acceleration was $150,000 \mathrm{rad} / \mathrm{s}^{2}$. Such accelerations typically lead to several tens of revolutions per second for large rounds. As is the case with all of the acceleration figures here, Froude scaling principles hold when arriving at estimates for smaller (or larger) rounds, which indicates that lower caliber rounds will encounter acceleration levels as the reciprocal of the scale factor. It will be seen that although spinning passive rounds is a good way to provide stabilization to otherwise unstable, unguided shells, high rotational accelerations are extremely detrimental to actuators and control effectors are complicated substantially by high spin rates. Accordingly, most GNC packages which are shot today on controlled rounds and planned for the future are despun either by decoupling them from a spinning body or not spinning the body at all via an obturating band or the use of a smooth bore gun barrel.

\subsection{Thermal Environment}

From Ref. $16,17, \& 19$, it can be seen that minimum operational and storage temperatures have a strong influence on the design of the actuator elements themselves as they rely upon coefficient of thermal expansion mismatch to precompress actuator elements. Precompression levels at depressed temperatures must be carefully matched to thickness ratios and launch accelerations to ensure actuator survival of setback accelerations. Another consideration of great importance is thermal variation of $d_{31}$ and $d_{33}$ at depressed temperatures. Many investigators have made actuator elements and control devices which function just fine at room temperature, but will essentially die if exposed to a tactical environment. Accordingly, proper actuator material selection must be made with strong consideration of the operational and storage temperatures. Ref. 25 lists temperature environments which are typical of many military munitions as:

$$
-40^{\circ} \mathrm{C}\left(-40^{\circ} \mathrm{F}\right) \text { to }+63^{\circ} \mathrm{C}\left(145^{\circ} \mathrm{F}\right) \text { storage } \quad-9^{\circ} \mathrm{C}\left(+15^{\circ} \mathrm{F}\right) \text { to }+63^{\circ} \mathrm{C}\left(145^{\circ} \mathrm{F}\right) \text { operational }
$$

\subsection{Storage and Lifespan}

Storage and lifespan considerations also have a strong effect on round and adaptive actuator design because many grades of adaptive materials age considerably over long periods of time. References 27 and 28 list 20 years of controlled storage life which is typical for most munitions. Uncontrolled storage requirements vary widely from system to system, but typically range from 1 to 10 years. ${ }^{28}$

\subsection{Power Management, Consumption and Delivery}

Because all actuator systems will consume electrical energy, power management is a big issue in guided munitions. 20 year storage requirements and comparatively high voltages which are used with piezoelectric actuators complicate battery systems; accordingly, advanced capattery systems have been the power supply system of choice for most modern adaptive munitions. ${ }^{20-22}$

\subsection{Bandwidth}

Bandwidth is an important parameter for the design of any flight control system. For spinning guided munitions, the lowest control frequencies for achieving full pitch and yaw control is of course $1 / \mathrm{rev}$. Because most guided hard-launch munitions are despun, aeromechanics drives actuator requirements to control all fundamental modes. Because the longitudinal (and directional) short period modes have the highest frequency contents, flight control system designers typically fix designs around them. Over the past 9 years of research, it has been discovered that it is desirable to have actuator system corner frequencies at least $4 \mathrm{x}$ greater than the short period body modes to achieve high hit probabilities. ${ }^{16,17,19-22}$ Such frequencies range from $100+\mathrm{rad} / \mathrm{s}$ on larger caliber projectiles to over $1,000 \mathrm{rad} / \mathrm{s}$ on bullets. ${ }^{19-22}$

\subsection{Shipset Costs}

Some of the principal factors which drive material selection and actuator configurations are the shipset, system and life cycle costs. Because many systems are highly cost sensitive, guidance and actuator systems should be kept at least 
one order of magnitude below the target shipset cost for viability. One of the more modern systems which can provide a rough reference for guided munitions is the Mk171 ERGM which is projected to run between $\$ 35,000$ and $\$ 65,000$ each with guidance electronics packages ranging from $\$ 5,000-\$ 7,000 .^{26,29}$ These costs are contrasted against conventional "cargo" munitions of similar caliber which cost between $\$ 1,600$ and $\$ 2,200$ per round. ${ }^{29}$ Accordingly, the cost growth between guided and unguided variants of individual rounds is one to two orders of magnitude. If one examines the current US Army inventory, there is a spread of at least one order of magnitude between minimum and maximum cost per conventional round. This is typically influenced by lot size and functionality of the shell, so the trendlines are not perfectly straight; nonetheless, general acquisition cost trends can be seen in Figure 2.

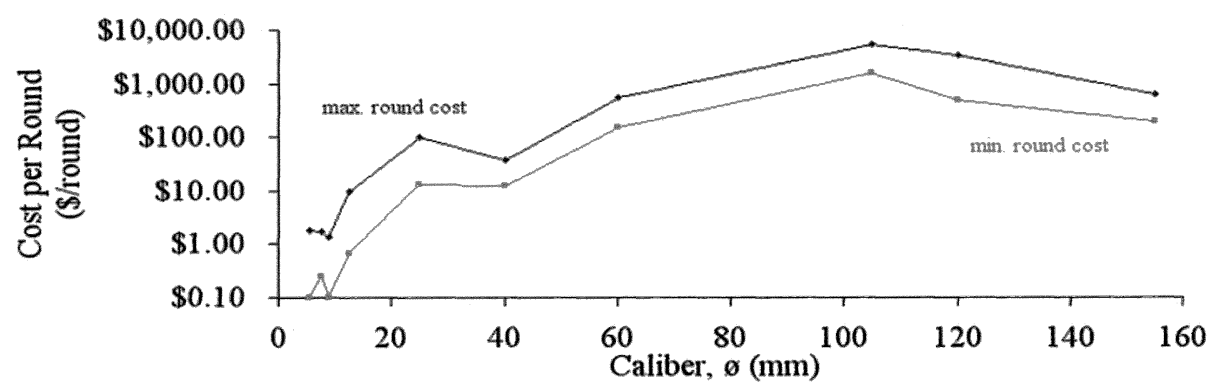

Fig. 2 Maximum \& minimum acquisition cost data for unguided US Army ammunition, $2002-2005^{30}$

By using the one-to-two orders of magnitude rule of thumb in cost growth per round, a guided munitions designer can have at least a rough estimate of acceptable cost levels. From Figure 2, it can be seen that a cost ceiling on the order of $\$ 10$ - $\$ 100 /$ round will box in the lowest caliber designs of guided bullets. Accordingly, it is painfully clear that only the most elementary guidance, navigation and control schemes can be used on such rounds to meet such conditions. It is not surprising that low caliber guided munitions typically call out flat trajectory, line-of-sight guidance schemes to keep rounds simple and costs low. Although larger caliber rounds enjoy more cost growth freedom, their extended range, need for GPS and/or inertial guidance and larger sizes also pose considerable challenges to the designer.

\subsection{Control Authority Requirements}

The amount of control authority required of flight control actuators is a strong function of mission, round design, effector layout and prevailing flight conditions. Currently, adaptive rounds have been designed to generate from just over 1 to more than 50g's of maneuver acceleration. Rounds with low levels of maneuver authority are typically used for sniper or direct fire missions. To the uninitiated, it may seem that atmospherics dominate actuator requirements at extended range; however, it is actually the flat trajectory requirement which typically sets flight control system designs and limits. ${ }^{20,21}$ As the round travels downrange, inexpensive, robust, line-of-sight guidance packages typically command flat trajectories, which means that the round must maneuver with at least $1 \mathrm{~g}$ of authority simply to resist the pull of gravity. Accordingly, the comparatively low dynamic pressures at extended ranges often make maintenance of lift equilibrium more challenging than even resisting a $99^{\text {th }}$ percentile gust. References 20 and 21 show that the current crop of conventional, inexpensive stoichiometries of piezoceramic actuator materials like PZT-5A can be successfully used for full flight control with inplane actuator free-strain levels of only $200 \mu$ strain.

On the other side of the spectrum are highly maneuverable intercepting rounds. These are designed to engage targets with 50g's or more of maneuver authority. Such round configurations are only remotely similar to the low authority designs. The most profound differences are in the external configuration, actuator material stoichiometries and flight control scheme. Ref. 22 shows that only extremely high performance single crystal piezoelectric elements can successfully steer such high control authority rounds via conventional flight control actuator configurations. Although the actuators can produce more than 6,000 $\mu$ strain of in-plane, free-element strain with good stiffness properties, their extremely high acquisition cost makes them ill-suited to the mission. Indeed, considering a simple $23 \mathrm{~mm}$ round, the cost of the raw actuator material alone exceeds $\$ 23,000$ per shipset in laboratory lots. ${ }^{22}$ Although it is estimated that high lot buys will drop this cost by an order of magnitude, the cost would still be a major departure from the costing targets above. Fortunately, advanced actuator designs are under development which allow low-cost piezoceramic actuators to achieve rate, force and control surface deflections which were formerly only achievable with expensive single-crystal elements. ${ }^{23}$ These actuators have been designed with the same level of control authority, force and bandwidth as the single-crystal actuators, but the raw materials cost only $\$ 11.30$ in laboratory lots for the same $23 \mathrm{~mm}$ system. 


\section{GENERALIZED ACTUATOR CONFIGURATION AND DESIGN FUNDAMENTALS}

\subsection{General Actuator Tendon Geometry}

From Figure 1, it can be seen that piezoelectric tendon actuator designs are principally oriented along the longitudinal axis of the round. This is of course done to maximize the resistance to setback and setforward accelerations. Cant angles of $10^{\circ}$ or less from the round centerline have been shown to be acceptable if properly supported. Figure 3, below, shows the most generic arrangement of a typical hard-launch capable flight control actuator element.

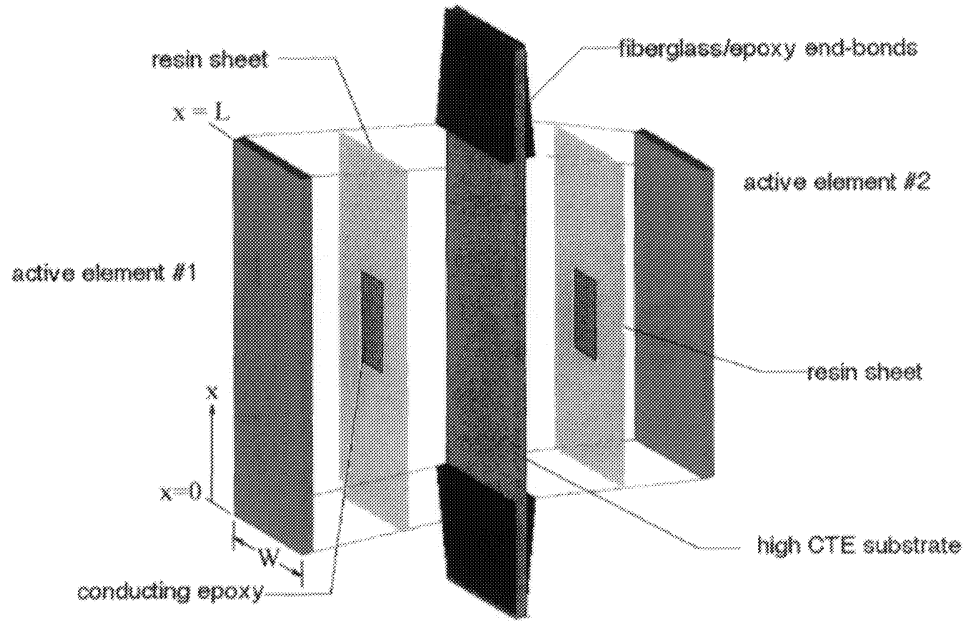

Fig. 3 General configuration of a typical hard-launch tolerant adaptive actuator element

\subsection{General Actuator Tendon Material Selection and Class I Design Procedures}

Although several different stoichiometries of adaptive materials have been used as the active elements, the most common for cost-sensitive rounds has been PZT-5A because of its good temperature stability, active strain generation capability and amenability to mass production. If no cost constraints are present, single-crystal piezoceramics are typically called out. A rough sketch of the initial design procedure for matching selected materials to cure temperatures and actuator lengths of rectanguloid tendon actuators follows:

i. Obtain setback, setforward and balloting accelerations and minimum operational temperature

ii. Obtain required active strain levels for given mission

iii. Obtain all material properties for substrates and actuators under consideration

iv. Use modeling techniques below to set the initial cure temperatures and actuator length

Two major loads will present themselves to the actuator element. These are axial and perpendicular imposed body forces due to setback, setforward and rotational accelerations. Because it can easily be shown that the launch loads which are expected (up to 100,000 g's of setback) will simply shear any actuator lamina if the actuator is not aligned properly, this loading case will not be considered in this basic analysis. It should be noted that these shear loads are typically introduced by rotational accelerations due to rifling. which can be dealt with by launching from a smooth bore gun with a sabot. Accordingly, the PZT actuators should be placed directly along the axis of acceleration and supported in tendon actuation bays. If this loading is present, and gravity forces are neglected, then an expression for load along the body longitudinal axis of a rectangular element is obtained during launch:

$$
F(x)=n g\left[x\left(\rho_{a} A_{a}+\rho_{s} A_{s}\right)+m_{o}\right]-F_{o}
$$

Where $F_{0}$ is an applied force on the end of the element from the base support, $n$ is the amount of acceleration, $g$ is the acceleration of gravity, $x$ is the longitudinal dimension of the actuator from the free end forward, $\rho_{a}$ and $\rho_{s}$ are the actuator, substrate densities respectively and $A_{a}$ and $A_{s}$ are the areas and $m_{o}$ is an end fixture mass.

If the laminate is assumed to be balanced and symmetric and both PZT actuators are energized in phase, then a laminate extension in two directions will occur. This active force combined with thermally induced stresses and laminate resistive forces yields an expression for overall force balance at any given point in the unloaded laminate. 


$$
\left(E_{a} A_{a}+E_{s} A_{s}\right) \varepsilon=\left(E_{a} A_{a} \alpha_{a}+E_{s} A_{s} \alpha_{s}\right) \Delta T+E_{a} A_{a} \Lambda
$$

Where $\mathrm{E}$ is the stiffness of the individual constituents, $\alpha$ is the coefficient of thermal expansion and $\Lambda$ is the free expansion strain of the actuator. This precompression may be introduced through the use of a favorable coefficient of thermal expansion mismatch between the substrate and piezoelectric actuator element.

Because it can easily be seen that the stress upon launch is maximized at the top of the actuator which is critical in tension, equations 3 and 4 may be combined to determine the stress level at $\mathrm{x}$ for actuators of rectanguloid configuration, counting $\mathrm{x}$ as distance from the bottom of the actuator where end mass, $m_{0}$ is attached and force $F_{o}$ is applied.

$$
\left(E_{a} A_{a}+E_{s} A_{s}\right) \varepsilon=\left(E_{a} A_{a} \alpha_{a}+E_{s} A_{s} \alpha_{s}\right) \Delta T+E_{a} A_{a} \Lambda+n g\left[x\left(\rho_{a} A_{a}+\rho_{s} A_{s}\right)+m_{o}\right]-F_{o}
$$

From Ref. 19, substrates like aluminum are shown to have been chosen because they possess significantly higher coefficients of thermal expansion than the PZT actuators. This is significant because an elevated temperature cure will effectively precompress the elements following resin set and cool-down and make them more robust. It should be noted at this point that graphite/epoxy composites were not considered as a suitable substrate material because they would place the PZT actuators in tension after an elevated temperature cure. (Such pre-tensioning is extremely detrimental to actuator performance and has been responsible for numerous actuator element failures in many parts of the adaptive structures industry.) If it is assumed that the laminate is unloaded and constrained flat during the cure which is below the Curie temperature of the PZT, then the total laminate strain may be solved, where $\Delta \mathrm{T}$ is the operational temperature - cure temperature (i.e. typically a negative value):

$$
\varepsilon=\frac{\left(E_{a} A_{a} \alpha_{a}+E_{s} A_{s} \alpha_{s}\right) \Delta T}{E_{a} A_{a}+E_{s} A_{s}}=\frac{\left(\alpha_{a}+\psi \alpha_{s}\right) \Delta T}{1+\psi}
$$

Following an elevated temperature cure, the PZT will be placed in compression while the substrate will be placed in tension. Expressions for pre-compression and pre-tensioning limitations are given as:

$$
\begin{aligned}
& \varepsilon_{c a p}=\varepsilon-\alpha_{a} \Delta T=\frac{\alpha_{a}+\psi \alpha_{s}}{1+\psi} \Delta T-\alpha_{a} \Delta T=\frac{\alpha_{s}-\alpha_{a}}{1+\psi} \psi \Delta T \\
& \varepsilon_{t s p}=\varepsilon-\alpha_{s} \Delta T=\frac{\alpha_{a}+\psi \alpha_{s}}{1+\psi} \Delta T-\alpha_{s} \Delta T=\frac{\alpha_{a}-\alpha_{s}}{1+\psi} \Delta T
\end{aligned}
$$

If one considers an actuator composite of variable cross-sectional area as a function of length, along with an end mass, $m_{0}$ which pulls on the actuator end with increased setback accelerations and end force, $F_{0}$, tends to push up and support the actuator at the free end, then the passive strain at any given location of the actuator can be solved for:

$$
\varepsilon_{t s y}-\varepsilon_{t s p} \geq \frac{n g\left(\int_{0}^{x}\left(\rho_{a} A_{a}(x)+\rho_{s} A_{s}(x)\right) d x+m_{o}\right)-F_{o}}{\left(E_{a} A_{a}(x)+E_{s} A_{s}(x)\right)}
$$

Because the maximum pretension strains will occur in the substrate at minimum operational temperatures, the substrate pretension strain, $\varepsilon_{\mathrm{tsp}}$, is fixed by the design condition at the lower temperature bound as follows:

$$
\varepsilon_{t s p}=\frac{\alpha_{a}-\alpha_{s}}{1+\psi} \Delta T_{\text {mintemp }}
$$


If the local width of the actuator and the substrate are assumed to be identical and the thickness ratio, $T=t_{s} / t_{a}$ is assumed to be constant over the length of the actuator (a good assumption for maintenance of low manufacturing costs), then, the load-matched width can be solved for as a function of length, strain limits and inertial terms:

$$
W(x)=\frac{n g m_{o}-F_{o}}{t_{a}\left(\varepsilon_{t s y}-\varepsilon_{t s p}\right)\left(E_{a}+T E_{s}\right)} e^{\frac{n g\left(\rho_{a}+T \rho_{s}\right) x}{\left(\varepsilon_{t s y}-\varepsilon_{t s p}\right)\left(E_{a}+T E_{s}\right)}}
$$

Equation 11 of course assumes that airloads on the actuator in free flight can be satisfied by the minimum actuator cross-sectional area which will occur at $x=0$. Often, other boundary conditions such dynamic response, end fixture mass or tendon tension for maintenance of structural connectivity are used to set the base width of the actuator. Mass and form factor constraints typically limit the end width of the actuator, $\mathrm{W}_{\mathrm{L}}$ which results in a more generalized expression for the width of the load-matched actuator design:

$$
W(x)=W_{o}\left(W_{L} / W_{o}\right)^{x / L}
$$

Equations 11 and 12 are contrasted against the solution for a rectangular actuator (constant width), which is the general configuration for most of the adaptive actuators which have been used so far (Ref. 19-23):

$$
W(x)=\frac{n g m_{o}-F_{o}}{t_{a}\left[\left(\varepsilon_{t s y}-\varepsilon_{t s p}\right)\left(E_{a}+T E_{s}\right)-n g l\left(\rho_{a}+T \rho_{s}\right)\right]}
$$

\section{PROOF-OF-PRINCIPLE ACTUATOR DESIGN, FABRICATION AND TESTING}

To demonstrate the utility of the Load-Matched designs, a series of experiments were performed on Load-Matched specimens and compared to conventionally designed actuators to determine load-carrying capacity and failure modes. For an initial starting point, it was assumed that a design space of $100 \mathrm{~mm}$ long $x 3 \mathrm{~mm}$ in diameter (typical of several classes of hard-launch munitions) would be considered.

\subsection{Test Specimens Design}

Given the above mentioned design space, and drawing upon the experiences of previous studies, a good choice of substrate material is 1100-H19 full-hard aluminum foil. Considering two actuator designs for comparison, one rectangular, one Load-Matched, using equation 12 the specifics of each are as follows:

Table 1 Rectangular and Load-Matched actuator constituent materials and geometries

\begin{tabular}{|l|l|l|}
\hline Rectangular Actuator $\mathrm{W}(\mathrm{x})=1.85 \mathrm{~mm}$ & \multicolumn{2}{|c|}{} \\
\cline { 1 - 2 } Load-Matched Actuator: $\mathrm{W}(\mathrm{x})=3^{(\mathrm{x} / 100)} \mathrm{mm}$ & active material & substrate \\
\hline $\mathrm{L}=100 \mathrm{~mm}$ & PZT-5A piezoceramic & $1100-\mathrm{H} 19$ full hard aluminum \\
\hline $\mathrm{m}_{\mathrm{o}}=30 \mathrm{mg}$ & $\mathrm{t}_{\mathrm{a}}=381 \mu \mathrm{m}$ & $\mathrm{t}_{\mathrm{s}}=38.1 \mu \mathrm{m}$ \\
\hline $\mathrm{m}_{\text {tot }}=600 \mathrm{mg}$ & $\mathrm{E}_{\mathrm{a}}=63 \mathrm{GPa}$ & $\mathrm{E}_{\mathrm{s}}=70 \mathrm{GPa}$ \\
\hline $\mathrm{T}_{\text {cure }}=160^{\circ} \mathrm{C}$ & $\varepsilon_{\mathrm{tlim}}=300 \mu \mathrm{strain}$ & $\varepsilon_{\mathrm{ty}}=2950 \mu \mathrm{strain}$ \\
\hline$\Delta \mathrm{T}=-140^{\circ} \mathrm{C}$ & $\rho_{\mathrm{a}}=7.83 \mathrm{~g} / \mathrm{cc}$ & $\rho_{\mathrm{s}}=2.70 \mathrm{~g} / \mathrm{cc}$ \\
\hline $\mathrm{T}=0.1$ & $\alpha_{\mathrm{a}}=4.5 \mu \mathrm{strain} /{ }^{\circ} \mathrm{C}$ & $\alpha_{\mathrm{s}}=23.5 \mu \mathrm{strain} /{ }^{\circ} \mathrm{C}$ \\
\hline$\psi=0.111$ & & \\
\hline
\end{tabular}

One will note that a general tensile strain limit which has been experimentally observed for hard-launch PZT-5A type piezoceramics is $\varepsilon_{\text {tlim }}=300 \mu$ strain for maintenance of both structural integrity and poling. The tip mass of $\mathrm{m}_{\mathrm{o}}=30 \mathrm{mg}$ is representative of end bonds and control effector flexures. Accordingly, if one uses two 190.5 $\mu \mathrm{m}$ (7.5mil) thick PZT-5A sheets laminated on either side of a $0.0381 \mathrm{~mm}(1.5 \mathrm{mil}) 1100-\mathrm{H} 19$ aluminum substrate, both the thickness of the substrate and the cure temperature can be matched in the rectangular and Load-Matched actuator designs. Figure 4 shows the strain profile in the two actuators and the design boundaries. 


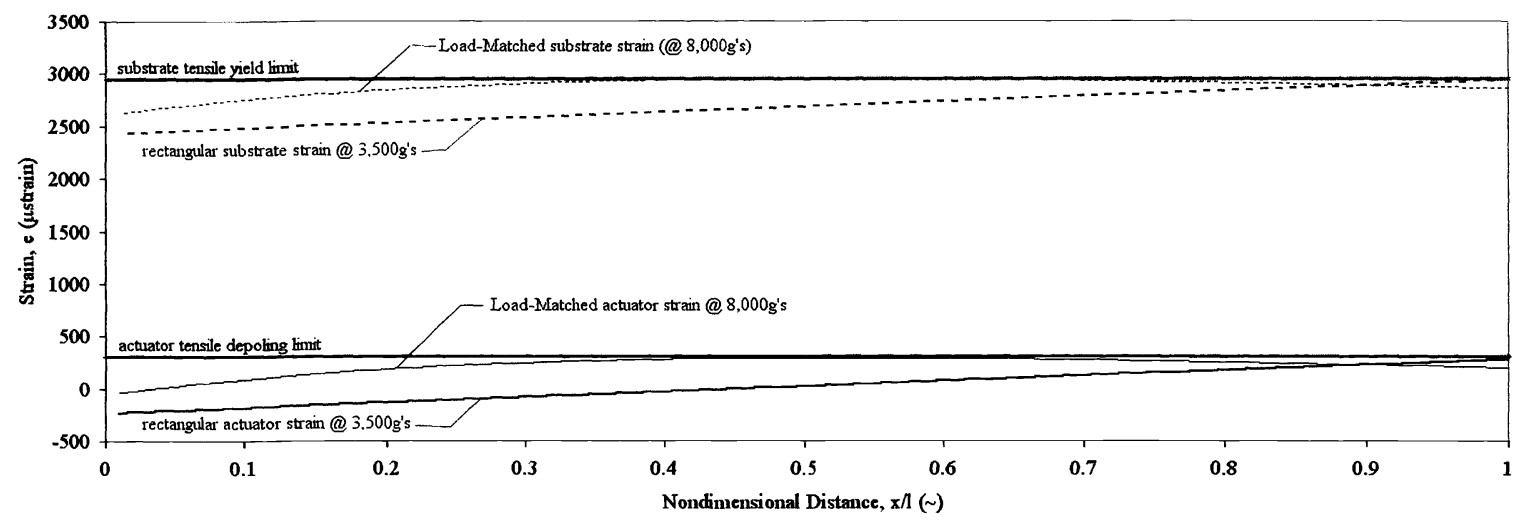

Fig. 4 Strain distributions in 100mm long, 600mg rectangular and Load-Matched actuators at 3,500 and 8,000 g's of setback acceleration

Although Figure 4 shows both the rectangular and Load-Matched designs just meet both the actuator and substrate tensile limits, the rectangular actuator element reached those limits at only 3,500 g's of acceleration while the LoadMatched actuator was designed to $8,000 \mathrm{~g}$ 's.

\subsection{Test Specimen Fabrication}

Ten of each type of specimen were fabricated from PZT-5A and 1100-19H aluminum for testing and built to the geometries specified in Table 1 and Figure 3 . The piezoceramic sheets and aluminum substrates were buffed to 1000 grit finish, cleaned and treated with 0.5 molar HCL prior to bonding. The PZT-5A sheets were bonded to the surface with a 15 $-25 \mu \mathrm{m}$ thick layer of Scotchweld epoxy tape and MasterBond EP21TDC-N conducting epoxy. End fixtures were fabricated from two $10 \mathrm{~mm}$ end-runs of style 120 fiberglass cloth bonded and imbibed with Scotchweld epoxy, leading to an overall test specimen length of $120 \mathrm{~mm}$. The active lamina were cured under $2 \mathrm{~atm}$. of pressure at $160^{\circ} \mathrm{C}$ for two hours. Following the cure, each actuator was deflashed, trimmed and deburred to a 1600 grit end polish to eliminate stress risers. The edges of the piezoceramic sheets were trimmed back and rounded to $50 \mu \mathrm{m}$ radius to eliminate lateral stress peaks during activation. Polarity, continuity, impedance and actuation characteristics were checked with removable electrodes following post processing. Figure 5 shows two of the twenty actuator elements which were fabricated for the test sequence. Following experimental verification of actuator performance, balancing masses were bonded with cyanoacrylate resin to the actuator tips so as to generate loads equal to the $30 \mathrm{mg}$ fixtures found in flightworthy rounds.

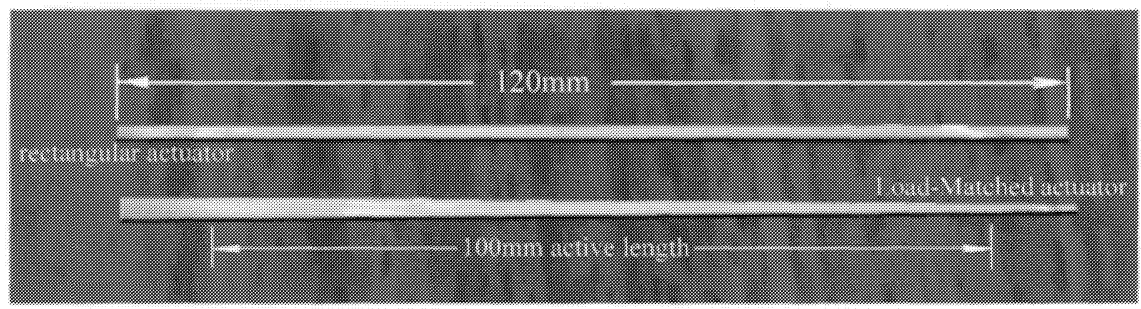

Fig. 5 Rectangular and Load-Matched PZT-5H/1100-19H aluminum actuator test specimens

\subsection{Test Set-Up and Procedures}

The test apparatus was designed to impart longitudinal loads primarily emulating setback accelerations, while constraining the specimen laterally so as to emulate the confines of the actuator bays. Accordingly, the actuators were laminated between a pair of $25 \mu \mathrm{m}$ polyester sheets which were in turn bonded to the insides of the test arch. Figure 6 shows the general arrangement of the test arch with the specimen mounted for testing. 

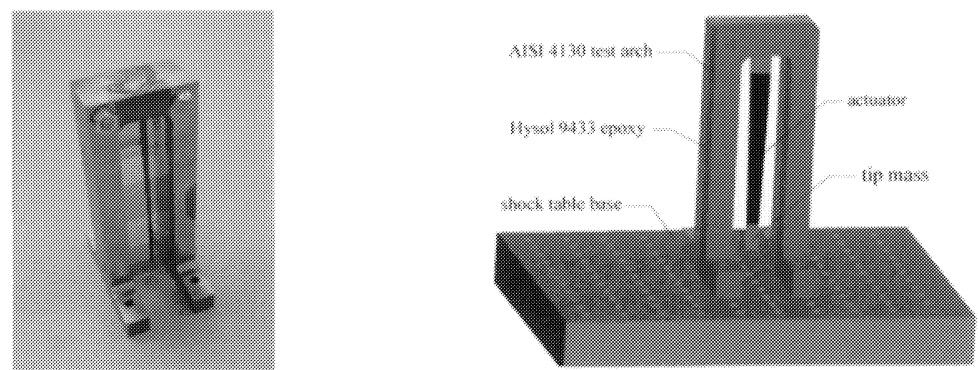

Figure 6 Typical actuator mounting arrangement in the steel test arch and fixture of the test arch to the shock table base

Shock testing was conducted on a vertical shock table assembly with acceleration data collected at 20,000 samples per second as measured via piezoelectric accelerometers. Figure 7 shows a typical acceleration profile for a $10,081 \mathrm{~g}$ impulse. The reader is asked to note the magnitude and sign of the profile which indicates that several thousand g's of setforward acceleration is typically experienced during testing. Following shock testing, each specimen was tested for mechanical continuity, level and direction of poling.

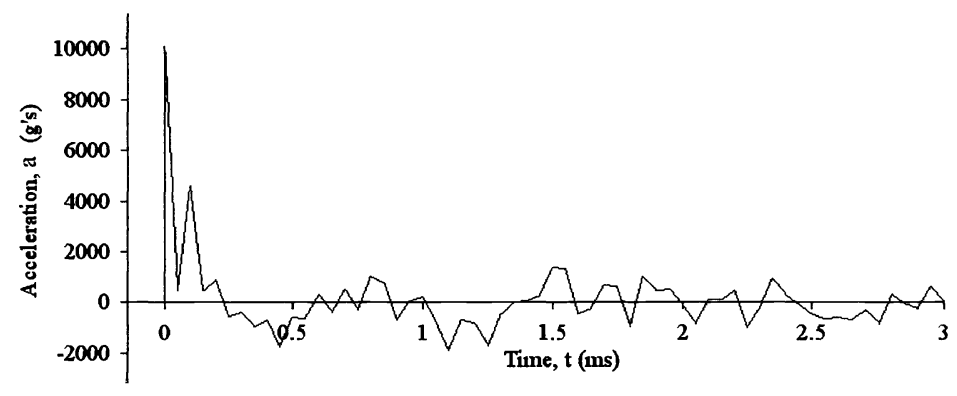

Fig. 7 Typical acceleration profile from shock-table testing $(10,081 \mathrm{~g}$ 's)

\subsection{Test Results}

The test results showed two dramatic sets of data which demonstrated that the static analysis is a conservative estimate of failure acceleration levels. Figure 8 shows that both the rectangular and Load-Matched actuator designs did significantly better than predicted by static analysis. The rectangular actuators failed between 3,833 and 3,931g's of acceleration (between specimens 6 and 7) which is 9.5 and $12 \%$ above the level predicted by static analysis. Load-Matched specimens failed between 9,377 and 9,670g's of acceleration (between specimens 15 and 18 ) which is 17 and $21 \%$ above the level predicted by static analysis. Figure 8 shows that specimen 17 (9,567g's) endured more acceleration than 16 $(9,411 \mathrm{~g}$ 's). Even though static stress prediction showed the actuator ends to be less loaded than the middle, it is thought the reasons why all specimens failed at the end bonds is because of the cyclical cold-work done on the substrate following the primary acceleration pulse. Post shot testing showed that all of the specimens that survived mechanically demonstrated no measurable loss in $\mathrm{d}_{31}$ or other piezoelectric properties.

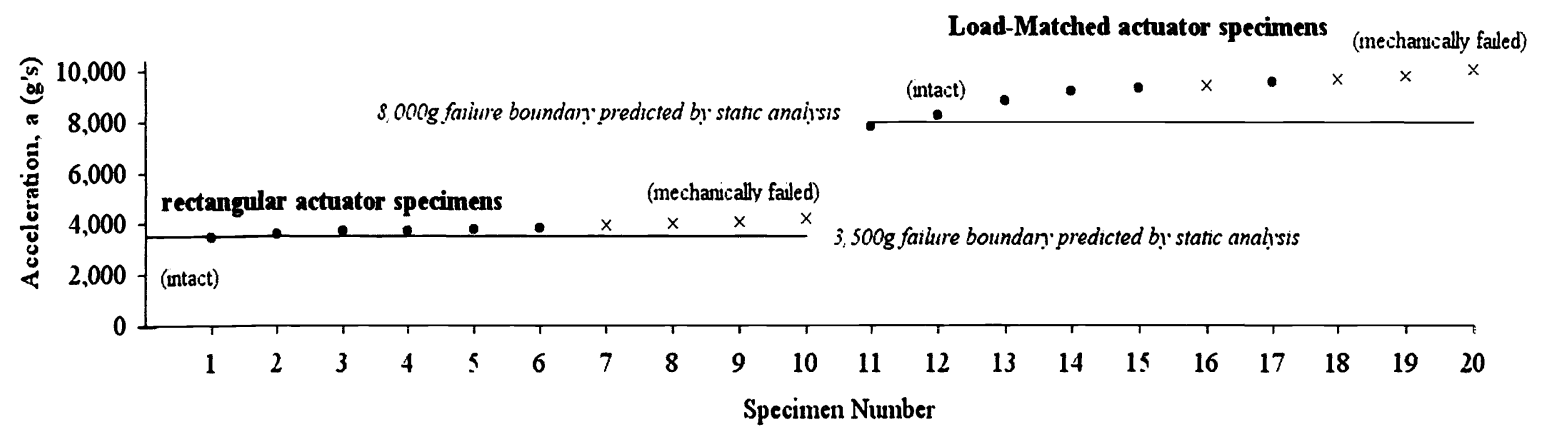

Fig. 8 Rectangular and Load-Matched actuator acceleration failure levels 


\section{CONCLUSIONS}

It can be concluded that laminated piezoelectric actuators can be successfully fabricated so as to withstand high-g shock environments typically experienced by hard launched munitions through the appropriate use of thermal expansion mismatch techniques. These techniques employ balanced active materials bonded to substrates which possess much higher CTE's than the active materials. It has been shown that the active materials in such lamina may be precompressed to significant levels, thereby lending a high degree of resistance to depoling and mechanical failure as the elements are subjected to tensile shock loading. A basic technique for matching design strains within the actuators to local actuator width demonstrates that an exponential form factor leads to a more favorable strain distribution in such active elements rather than is seen in rectangularly shaped actuators.

To demonstrate this concept, two families of active actuators were designed and fabricated with the same mass and length. The first actuators tested had a mass of $600 \mathrm{mg}$, were $100 \mathrm{~mm}$ long, rectangular in shape and composed of two $190.5 \mu \mathrm{m}$ PZT-5A piezoceramic sheets laminated to a $38.1 \mu \mathrm{m}$ substrate of $1100-19 \mathrm{H}$ full hard aluminum foil and cut to $1.85 \mathrm{~mm}$ width. The second family of Load-Matched actuators had all of the same characteristics as the first, except that they employed an exponential profile which gave them a base width of $3 \mathrm{~mm}$ and a tip width of $1 \mathrm{~mm}$. Both families of actuator elements were fitted with tip masses of $30 \mathrm{mg}$ so as to emulate end fixtures typically found in hard-launched munitions of this size. Static analysis techniques were used to set the cure temperature at $160^{\circ} \mathrm{C}$ so that substrate and active material tension limits would be reached at exactly the design condition which was $3,500 \mathrm{~g}$ 's for rectangular elements while the Load-Matched actuators reached 8,000g's. Two groups of 10 specimens were prepared and shock table tested, showing that the rectangular specimens failed at acceleration levels $9.5-12 \%$ over the predicted failure level $(3,833$ to $3,931 \mathrm{~g}$ 's $)$. The Load-Matched actuators failed between 17 and $21 \%$ above the predicted failure acceleration level of $8.000 \mathrm{~g}$ 's $(9,377$ and $9,670 \mathrm{~g}$ 's), thus clearly demonstrating that adaptive actuators for hard-launched munitions can be made nearly 2.5 times more acceleration tolerant if appropriate compression and geometric tailoring techniques are employed.

\section{ACKNOWLEDGMENTS}

The authors wish to acknowledge the Technical University of Delft Faculty of Aerospace Engineering for supporting this effort.

\section{REFERENCES}

1. Pike, John, "Low Cost Competent Munitions (LCCM)," published by Global Security.org, Alexandria, Virginia, October, 2002. (http://www.globalsecurity.org/military/systems/munitions/cm-gps.htm)

2. Linder, Doug, Huiyu Zhu, Diann Brei and Jim Vindlinski, "Inertially Stabilized Rifle Using Recurve Actuators," proceedings of IMECE '02, ASME International Mechanical Engineering Congress and Exposition, New Orleans, Louisiana, November 17 - 22, 2002, paper IMECE2002-33987.

3. Pike, John, "M712 Copperhead," published by Global Security.org, Alexandria, Virginia, October, 2002. (http://www.globalsecurity.org/military/systems/munitions/m712.htm)

4. Pike, John, "XM982 Excalibur Precision Guided Extended Range Artillery Projectile," published by Global Security.org, Alexandria, Virginia, October, 2002. (http://www.globalsecurity.org/military/systems/munitions/m982155.htm)

5. Pike, John, "M898 SADARM (Sense and Destroy Armor)" published by Global Security.org, Alexandria, Virginia, October, 2002. (http://www.globalsecurity.org/military/systems/munitions/sadarm.htm)

6. Parsch, Andreas, "Mk 171 Extended Range Guided Munition (ERGM)," Directory of U.S. Military Rockets and Missiles, published by Designation-Systems, Münich, Germany, 2003.

7. Reusch, Olaf, and Karl Kautzsch," Precision Enhancement build on a Multi-Functional Fuze for 155mm Artillery Munition," proceedings of the $47^{\text {th }}$ NDIA Annual Fuze conference, April, 2003. (http://www.dtic.mil/ndia/2003fuze/kautzsch.pdf)

8. Taylor, Alex, "Smart Mortar Ammunition - British Royal Ordnanace $81 \mathrm{~mm}$ Millimetric Radar Guided Round," published by Global Defence Review, Ltd., London, United Kingdom, 2000. (http://www.globaldefence.com/2000/pages/mortar.html)

9. Anon., "M247 Sergeant York DIVAD," published by the Federation of American Scientists, Washington, D.C., 1999. (http://www.fas.org/man/dod-101/sys/land/m247.htm) 
10. Barrett, R., "Active Plate and Missile Wing Development Using EDAP Elements," Journal of Smart Materials and Structures, Institute ofPhysics Publishing, Ltd., Techno House, Bristol, UK, Vol. 1, No. 3, pp. 214226, ISSN 096.

11. Barrett, R., "Active Composite Torque-Plate Fins for Subsonic Missiles," paper presented at the Dynamic Response of Composite Structures Conference, New Orleans, Louisiana, August 30 - September 1, 1993.

12. Barrett, R., "Active Plate and Missile Wing Development Using DAP Elements," AIAA Journal,March, 1994.

13. Barrett, R., "All-Moving Active Aerodynamic Surface Research," proceedings of the Society of Engineering Science 31st Annual Technical Meeting, Texas A\&M University, College Station, TX, October 10-12, 1994, published by the Institute of Physics Publishing, Ltd., Techno House, Bristol, UK, Vol. 4, No. 4, June 1995, pp. 65 - 74.

14. SCRAM Report Barrett, R., "Design, Construction and Testing of a Proof-of-Concept Smart Compressed Reversed Adaptive Munition," Final Report to the USAF Armament Directorate, Wright Laboratory, Eglin AFB, FL contract no. AF-FO8630-95-K-0079, September, 1996.

15. Barrett, R., "Construction and Test Report for the Rotationally Active Linear Actuator (RALA) Adaptive Canard, Final Test Report for McDonnell Douglas Aerospace/Boeing Aircraft Company Advanced Missile Systems and Subsystem Applications, St. Louis, MO contract no. FO 8630-95-C-0009, August, 1997.

16. Barrett, R., "Invention and Evaluation of the Barrel Launched Adaptive Munition (BLAM)," final report for USAF contract no. F-49620-93-C-0063, USAF Wright Laboratory Flight Vehicles Branch, WL/MNAV August, 1995.

17. Barrett, R., and Stutts, J. "Barrel-Launched Adaptive Munition BLAM Experimental Round Research," final report for USAF contract no. F-49620-C-0063, subcontract 96-0869, USAF Wright Laboratory Flight Vehicles Branch, WL/MNAV February, 1997.

18. Winchenbach, G., "Cone Aerodynamics Test", Aeroballistic Research Facility Ballistic Spark Range Technical Report, USAF Wright Laboratory Flight Vehicles Branch, WL/MNAV June 1996.

19. Barrett, R. and Stutts, J., "Modeling, Design and Testing of a Barrel-Launched Adaptive Munition," proceedings of the 4" Annual Society of Photo-Optical Instrumentation Engineers Symposium on Smart Structures and Materials, San Diego, CA, 3-6 March 1997.

20. Lee, Gary, "Range-Extended Adaptive Munition (REAM)" Final Report from Lutronix Corporation to the Defense Advanced Research Projects Agency (DARPA), Del Mar, California, April 1999.

21. Lee, Gary, "40/50 Caliber Range-Extended Adaptive Munition (REAM)" Final Report from Lutronix Corporation to the US Army TACOM-ARDEC Del Mar, California, May 2000.

22. Lee, Gary, "Shipborne Countermeasure Range-Extended Adaptive Munition (SCREAM)," Final Report from Lutronix Corporation to the US Army TACOM-ARDEC, Del Mar, California, May 2003.

23. Barrett, R., and P. Tiso, "PBP Adaptive Actuators and Embodiments," International Patent Filing from the Faculty of Aerospace Engineering, Technical University of Delft, February 2004.

24. Anon., "Actual Results and Analysis," Hypervelocity Weapon System (HVWS) Field Experiment 1 Final Report, Volume 1 Technical Report, published by Technology Gateways, Inc., Niceville, Florida, 1993, p. IIIC-6.

25. Hertlein, Robert and Mark Miner, "Extended Range Guided Munition (ERGM) Safe and Arm Device and Height-ofBurst Sensor," paper presented at the NDIA Fuse Conference, 9 April 2993, New Orleans, LA. (http://www.dtic.mil/ndia/2003fuze/hertlien.pdf)

26. Erwin, Sandra, "Naval Guns: Can They Deliver “Affordable” Precision Strike?" National Defense Magazine, published by National Defense Industrial Association, Arlington, Virginia, March 2001. (http://nationaldefense.nkia.org/article.cfm?Id=441)

27. Pike, J. "Trajectory Correctable Munition (TCM)," published by Global Security.org, Alexandria, Virginia, October, 2002. (http://www.globalsecurity.org/military/systems/munitions/tcm.htm)

28. Anon., "M732A2 Proximity Fuse and M782 Multi-Option Fuse for Artillery (MOFA) Data Sheets," published by Alliant Techsystems, Inc. Edina, Minnesota, 2003. (http://www.atk.com/productsPrecision/descriptions/products/fuses/ artilleryfuzes.htm)

29. Engel, Mark, "Low Cost Guidance Electronics Unit (LCGEU)" proceedings of the $46^{\text {th }}$ Annual Fuze Conference, 29 April - 1 May 2002, published by the National Defense Industries Association, NDIA, Arlington, VA 2002.

30. Anon., "Precurement of Ammunition, Army," Department of the Army Procurement Programs, published by the US Army, Washington, D.C., 2003. (http://www.asafm.army.mil/budget/fybm/fy04-05/pforms/ammo.pdf) 